\title{
Regions at Risk in the Knee Joint of Young Professional Soccer Players: Longitudinal Evaluation of Early Cartilage Degeneration by Quantitative T2 Mapping in 3 T MRI
}

CARTILAGE

2021, Vol. I3(Suppl I) 595S-603S

(C) The Author(s) 2020

Article reuse guidelines:

sagepub.com/journals-permissions

DOI: 10.1 I77/1947603520924773

journals.sagepub.com/home/CAR

(SSAGE

\author{
Hanna Schenk', David Simon ${ }^{1,2}$, Leonie Waldenmeier ${ }^{3}$, Christoph Evers $^{4}$ iD, \\ Rolf Janka',5, Goetz H. Welsch', and Milena L. Pachowsky',2,7 iD
}

\begin{abstract}
Purpose. The study aims to detect regions at risk for (pre-)osteoarthritis in the tibiofemoral joint of young professional soccer players by evaluating cartilage composition by T2 mapping in a $3 \mathrm{~T}$ magnetic resonance imaging setting. Methods. In this longitudinal study, 20 professional adolescent soccer players were included. Tibiofemoral cartilage was assessed by quantitative T2 mapping and T2 values were evaluated by regions of interest analysis. Statistical evaluation, using Wilcoxon signed-rank tests, was performed to compare global T2 values and subregional T2 values between a baseline and a followup investigation 4.3 years later. Based on the average of playing time ( 15 years) we divided the cohort in 2 groups and differences were evaluated. Results. When comparing baseline and follow-up, our findings showed statistically significant increases of the global medial tibial and femoral T2 values. The most noticeable results of the subregional T2 analysis were statistically significant increases in the medial posterior zones (deep femoral 36.1 vs. $39.5, P=0.00 \mathrm{I}$; superficial femoral 57.0 vs. $62.4, P=0.034$; deep tibial 28.3 vs. $34 . I, P=0.009$; superficial tibial 43.2 vs. $55.3, P=0.002$ ). Conclusion. The elevation of T2 values in the medial, especially medial posterior, compartment of the knee joint indicates that these regions are at risk for early cartilage degeneration already at the time of adolescence. The findings can help individualize and optimize training concepts and to be aware of the chronic stress on these vulnerable areas. Prevention programs should be established in young players to avoid further cartilage damage.
\end{abstract}

\section{Keywords}

cartilage, MRI, T2 mapping, adolescent, sports

\section{Introduction}

Cartilage damage caused by traumatic sports injuries or due to overuse can affect the further life profoundly. Especially high-intensity sports like soccer are associated with a high risk for injuries. ${ }^{1}$ During this high-impact sport traumatic or overuse injuries at the lower extremities are common. ${ }^{1,2}$ Due to the high mechanical load there is a risk for cartilage damage in the knee joint of active athletes, which can progress to osteoarthritis $(\mathrm{OA})^{3}$ especially for professional soccer players. ${ }^{4,5}$ In weightbearing joints, knee OA is accelerated by local risk factors such as muscle deficit, obesity, and joint laxity with worse proprioception. ${ }^{6}$ Soccer players were younger than the non-athletes at the onset of the diagnosis. ${ }^{7}$ Currently available surgical or conservative treatment strategies were inadequate to prevent the development of OA. ${ }^{3,8-10}$ Therefore, the prevention of OA will have a positive impact on the quality of life of former soccer players. ${ }^{11}$ Most prior studies focused on middle-aged or
'Friedrich-Alexander University Erlangen-Nürnberg (FAU), Erlangen, Germany

${ }^{2}$ Department of Internal Medicine 3-Rheumatology and Immunology, University Hospital of Erlangen, Erlangen, Germany

${ }^{3}$ Department of Internal Medicine, University Hospital of Basel, Basel, Switzerland

${ }^{4}$ Department of Radiooncology, University Hospital of Halle/Saale, Halle, Germany

${ }^{5}$ Department of Radiology, University Hospital of Erlangen, Erlangen, Germany

${ }^{6}$ UKE Athleticum, University Hospital Hamburg-Eppendorf, Hamburg, Germany

${ }^{7}$ Department of Trauma and Orthopedic Surgery, University Hospital of Erlangen, Erlangen, Germany

\section{Corresponding Author:}

Milena L. Pachowsky, Department of Trauma and Orthopedic Surgery, University Hospital of Erlangen, Maximiliansplatz I, Erlangen 91054, Germany.

Email: milena.pachowsky@uk-erlangen.de 
retired football players. Investigations in adolescent soccer players will help develop programs to minimize early cartilage alterations. How can we identify the regions at risk in the knee joints of young athletes and would it be possible to individualize training and prevention programs? Modified training sections should be provided to minimize the high mechanical stress and to increase the regulatory mechanisms, such as the supporting muscle strength and the proprioceptive accuracy, in these vulnerable areas. Subtle evaluation with subregional analysis of the intraarticular structures is crucial for these aims. Standard magnetic resonance imaging (MRI) has become a well-established technique as a noninvasive morphological assessment of cartilage lesions. ${ }^{12}$ Recently, T2 mapping is gaining importance for quantitative evaluation of joint tissue, especially cartilage. ${ }^{13,14}$ The T2 relaxation times depend on tissue hydration and collagen orientation. ${ }^{15,16}$ Elevated T2 relaxation times reflect cartilage alterations. ${ }^{17-19}$

The primary objective of our longitudinal study is the qualitative and quantitative analysis of structural and biochemical changes in the knee cartilage of young professional soccer players using T2 mapping in a $3 \mathrm{~T}$ MRI setting. We hypothesized that the high mechanical load affects the cartilage structure during adolescence. It is conceivable that the detection of regions at risk can help to individualize training and prevention programs for players.

\section{Material and Methods}

The study protocol was approved by the local ethics committee and written informed consent was obtained from all subjects.

\section{Study Population}

For the longitudinal investigation, our study population was selected from a cohort of 57 male young professional soccer players. The volunteers were recruited from a youth academy of a professional soccer team of the first and second national leagues for multiple seasons. Players performed a high-frequency training 3 up to 5 times a week and took part in 1 or 2 matches per week in a professional soccer league in Germany. Soccer players with a break of playtime over 2 years between the baseline (B) and the follow-up (F) examination or an F period of more than 5.5 years or less than 3.5 years were excluded. Additionally, any injuries or pain of the lower extremities at the time of image acquisition and an irregular or reduced frequency of soccer practice caused by any traumatic knee lesion or a history of surgery in the past 6 months. Furthermore, players had to be excluded when they had joined a foreign soccer team or if they could not be reached by telephone. We included the remaining 20 players in our study population for the longitudinal investigation.
Table I. Parameters of the Magnetic Resonance Imaging Protocol.

\begin{tabular}{lc}
\hline Sequence & T2 Map \\
\hline Repetition time (ms) & 1300 \\
Echo times (ms) & $\mathrm{II} .9,23.8,35.7,47.6,59.5,7 \mathrm{I} .4$ \\
Field of view $(\mathrm{mm})$ & $0.2 \times 0.2 \times 3.0$ \\
Voxel size $(\mathrm{mm})$ & 20 \\
Distance factor $(\%)$ & 200 \\
Bandwidth (Hz/pixel) & 16 \\
Number of slices & 3 \\
Section thickness (mm) & 180 \\
Flip angle (deg) & $4: 28$ \\
Total acquisition time (min:s) & \\
\hline
\end{tabular}

All players have been actively playing soccer since their childhood and were regularly actively participating in training and matches at the time of observation.

The following data were retrieved from this homologous group on the examination days: age, weight, height, clinical parameters, further information of their soccer careers. Based on the duration of playing time, we divided the cohort in 2 groups: number of years playing soccer less than 15 years (group I) versus more than 15 years (group II).

The Lysholm score was documented.

\section{Image Acquisition}

For the $\mathrm{B}$ and the $\mathrm{F}$ examination a 3.0 T MR scanner (Magnetom Verio and Skyra, Siemens Medical Solutions, Erlangen, Germany) with a gradient strength of $45 \mathrm{mT} / \mathrm{m}$ and a 15-channel knee array coil (QED, Quality Electrodynamics, Mayfield Village, OH, USA) were used for the image acquisition. Analogous to the B investigation the non-dominant leg was selected, also described as supporting leg (left leg of all players). Prior to the MR examination, daily routine activities, but no soccer matches, were allowed. Patients were in a supine position while the images were acquired. The tightly fixed knee joint was fully extended and fixed in a neutral rotation position in the center of the coil. The T2 sequence was planned at the end of the MR examination after a few other sequences to represent unloading conditions to the cartilage. The mean time gap between the first sequence and the T2 sequence were $28.0 \pm 0.7$ minutes at the $\mathrm{B}$ examination and $27.1 \pm 1.1$ minutes at the $\mathrm{F}$ examination, this ensured comparable unloading conditions. A standardized image acquisition protocol was performed for both investigations. A high-resolution sagittal, multi-echo spin-echo sequence (MESE) using 6 echo-times for the quantitative T2 mapping was acquired. MRI parameters for the applied sequences are shown in Table 1. 


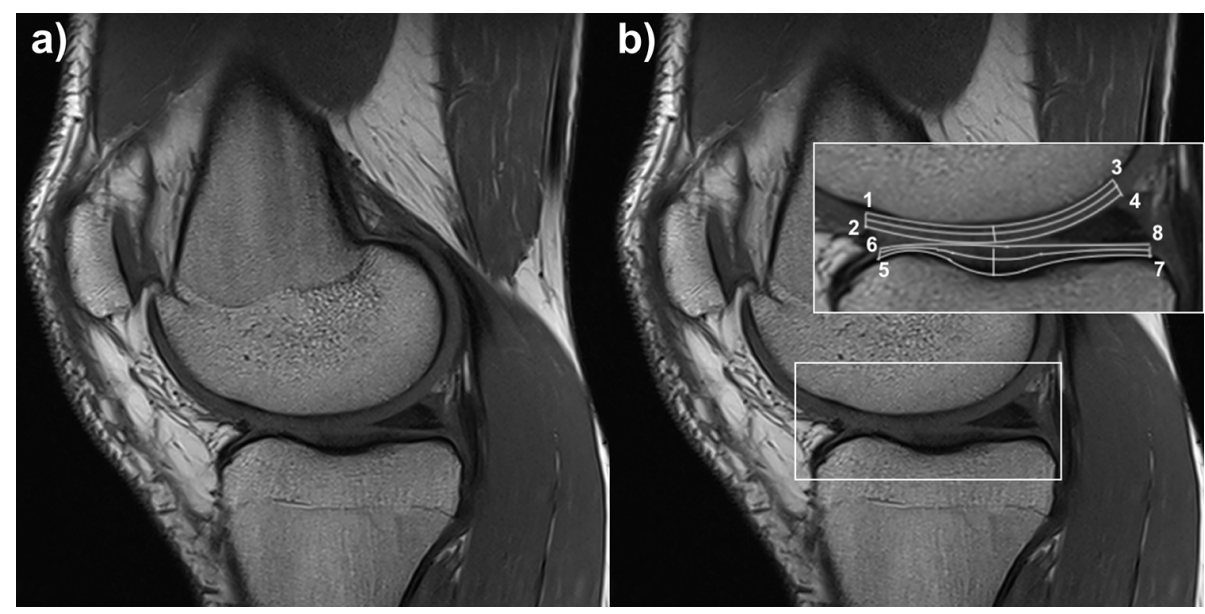

Figure I. Sagittal medial slice of a 19-year-old male soccer player, morphological image (a), schematic illustration of the regions of interest (ROIs) subdivision of the tibiofemoral cartilage (b). ROls were numbered as follows: I anterior deep femoral, 2 anterior superficial femoral, 3 posterior deep femoral, 4 posterior superficial femoral, 5 anterior deep tibial, 6 anterior superficial tibial, 7 posterior deep tibial, 8 posterior superficial tibial.

\section{Image Analysis}

T2 maps were obtained online via a pixel-wise, mono-exponential, nonnegative least squares (NNLS) fit analysis. The MRI data were transmitted to a Syngo (Leonardo) workstation (Siemens Medical Solution, Erlangen, Germany). First, the morphological data sets were examined for any circumscribed cartilage defects, cartilage thinning, motion artifacts or poor image quality. The regions of interest (ROI) were drawn manually by 2 junior experts ( 2 years of experience) under supervision of 2 senior experts (G.W. 15 years of experience, M.P. 10 years of experience) in musculoskeletal MRI. The ROIs were localized in the weightbearing zone of the tibiofemoral joint. To cover a big part of the weightbearing zone, the cartilage was assessed in 2 consecutive slices both in the medial and in the lateral joint compartment. On each slice, the cartilage was divided into 4 segments at the femur condyle (ROI 1-4) and 4 segments at the tibia plateau (ROI 5-8) for subregional analysis. A superficial (from the articular surface to the middle of the cartilage) and a deep zone (from the middle of the cartilage to the cartilage-bone interface) were defined. Then the cartilage was additionally divided into an anterior and a posterior zone. These zones were bordered by the peripheral margins of the menisci and the middle of the weightbearing zone. Figure 1 illustrates the ROI segmentation of the cartilage. Overall, 32 separate ROIs were analyzed on the tibiofemoral cartilage per patient per examination via T2 mapping. Besides, the single anatomical ROIs were combined to pooled ROIs (global medial and global lateral femoral cartilage, global medial and global lateral tibial cartilage). The pixel count of the ROIs showed a mean of $451.4 \pm 141.0$ (minimum 128 , maximum $925)$ in the B examination and a mean of $401.0 \pm 49.5$ (minimum 51, maximum 1157) in the F examination. For identical placement of the cartilage areas the same slices were used for the first and the second analysis. The same knee positioning within the coil and the section thickness of $3 \mathrm{~mm}$ ensured that the same cartilage areas were examined for the repeated measurements.

\section{Clinical Assessment}

The clinical outcome was evaluated by using the Lysholm score. It contains 8 subcriteria, 5 of which are subjective and 3 are functional. A total score of 100 points can be achieved. Effusion, range of motion and ligament stability were tested to check the clinical status of the supporting leg.

\section{Statistical Analysis}

Statistical analysis was performed using SPSS software (IBM SPSS 25.0 IBM Corp., Armonk, NY, USA). T2 values for all ROIs such as all demographic data (age, weight, height, body mass index, number of years playing soccer, Lysholm score) are given as mean \pm standard deviation.

The Kolmogorov-Smirnov test and the Shapiro-Wilk test did not confirm the assumptions of normally distributed continuous variables. The dependent $\mathrm{T} 2$ values were tested for differences between the $\mathrm{B}$ and the $\mathrm{F}$ examination for the pooled areas and the separate ROIs. These T2 relaxation times were evaluated by the Wilcoxon signed-rank test for the whole group and the 2 groups as defined above. For the inter- and intraobserver reproducibility, the intraclass correlation coefficient (ICC) was analyzed. $P$ values $\leq 0.05$ were considered as statistically significant. 
Table 2. T2 Relaxation Times (Mean $\pm \mathrm{SD}$ ) for All Single Anatomical Regions of Interest (ROls) of the Whole Group (N=19). ${ }^{\mathrm{a}}$

\begin{tabular}{llllll}
\hline Localization & & Baseline T2 (ms) & Follow-up T2 (ms) & $P$ Value Difference \\
\hline Medial & & & $37.2 \pm 7.7$ & $41.5 \pm 12.9$ & 0.159 \\
& ROI I & Anterior deep femoral & $56.0 \pm 8.2$ & $62.4 \pm 9.1$ & $\mathbf{0 . 0 1 6}$ \\
& ROI 2 & Anterior superficial femoral & $37.7 \pm 7.4$ & $39.4 \pm 7.9$ & 0.075 \\
& ROI 3 & Posterior deep femoral & $61.7 \pm 11.2$ & $65.0 \pm 11.0$ & 0.167 \\
& ROI 4 & Posterior superficial femoral & $34.4 \pm 7.6$ & $31.6 \pm 5.3$ & 0.275 \\
& ROI 5 & Anterior deep tibial & $46.9 \pm 7.6$ & $52.6 \pm 7.0$ & $\mathbf{0 . 0 1 8}$ \\
& ROI 6 & Anterior superficial tibial & $30.0 \pm 4.6$ & $33.8 \pm 4.6$ & $\mathbf{0 . 0 1 3}$ \\
Lateral & ROI 7 & Posterior deep tibial & $46.3 \pm 7.2$ & $54.5 \pm 1.1$ & $\mathbf{0 . 0 0 2}$ \\
& ROI 8 & Posterior superficial tibial & $34.0 \pm 9.5$ & $27.0 \pm 6.4$ & 0.00 \\
& ROI I & Anterior deep femoral & $48.9 \pm 10.2$ & $47.5 \pm 7.7$ & 0.104 \\
& ROI 2 & Anterior superficial femoral & $45.4 \pm 10.7$ & $49.2 \pm 11.0$ & $\mathbf{0 . 0 1 6}$ \\
& ROI 3 & Posterior deep femoral & $68.2 \pm 12.8$ & $75.9 \pm 13.2$ & 0.214 \\
& ROI 4 & Posterior superficial femoral & $27.5 \pm 4.7$ & $26.2 \pm 2.9$ & 0.065 \\
& ROI 5 & Anterior deep tibial & $38.9 \pm 8.5$ & $43.6 \pm 6.6$ & 0.258 \\
& ROI 6 & Anterior superficial tibial & $28.6 \pm 6.2$ & $30.5 \pm 7.4$ & $\mathbf{0 . 0 1 8}$ \\
& ROI 7 & Posterior deep tibial & $45.7 \pm 8.8$ & $53.0 \pm 11.9$
\end{tabular}

${ }^{a}$ The T2 values are displayed for the baseline (B) and the follow-up (F) examination with the calculated $P$ value for the difference. $P$ values $\leq 0.05$ are marked in boldface.

\section{Results}

\section{Patients Characteristics}

The F cohort consisting of 20 players had a mean age of $20.1 \pm 1.7$ years (range 18-23 years) and the body mass index was $23.2 \pm 1.3 \mathrm{~kg} / \mathrm{m}^{2}$ (range $20.5-25.2 \mathrm{~kg} / \mathrm{m}^{2}$ ).

The investigated volunteers started playing soccer at the mean age of 5.1 \pm 1.6 years (range 3-10 years) and joined the youth academy between the ages 6 and 17 years. On average, they had been playing soccer for $15.0 \pm 2.7$ years and thereafter $8.3 \pm 3.8$ years in the youth academy at the time of the $\mathrm{F}$ investigation. The time between the $\mathrm{B}$ and the F MR examination was 4.3 years with a mean $F$ period of 52 months (range 44-61 months).

The average of playing time ( 15 years) was our rationale for dichotomizing the cohort into groups. At the second examination, the following data were collected: 7 players had been playing soccer for less than 15 years (group I) and 12 players for more than 15 years (group II).

The T2 mapping images of 1 player of the F cohort had to be excluded because of poor image quality. Quantitative T2 mapping of the knee joint cartilage was assessed for the remaining 19 patients. A total of 1216 ROIs were analyzed.

\section{Image Analysis}

For a first overview, the pooled $\mathrm{T} 2$ values between the $\mathrm{B}$ and the $\mathrm{F}$ examination for the whole cohort were compared. An increase of the T2 values over time was detectable in the medial condyle. The T2 values in the weightbearing medial femoral zone were $48.2 \pm 6.3 \mathrm{~ms}$ during the first assessment and $52.2 \pm 7.9 \mathrm{~ms}$ during the second assessment. The difference was statistically significant $(P=0.026)$. The pooled $\mathrm{T} 2$ values in the medial tibial cartilage increased from $39.4 \pm$ 6.1 to $43.1 \pm 4.4 \mathrm{~ms}(P=0.032)$. No statistically significant differences in the lateral compartments were detected.

For the subregional analysis T2 values were compared according to the predefined ROIs. Statistically significantly higher $\mathrm{T} 2$ values in the $\mathrm{F}$ assessment were found in the following medial ROIs: ROIs 2, 6, 7, and 8. In the lateral ROIs of the knee joint a statistically significant increase of the T2 values was found in 2 of 10 zones: ROIs 4 and 8 . ROI 1 showed a statistically significant decrease of the T2 relaxation times. Table 2 presents these results.

Based on the duration of playing time we divided the cohort into 2 groups as defined above (group I: number of years playing soccer less than 15 years vs. group II: more than 15 years) and compared B with F T2 values.

When comparing B with $\mathrm{F}$ global $\mathrm{T} 2$ values in both groups, group II reached a statistically significant increase of global T2 times in the medial zones: weightbearing femoral cartilage $(45.7 \pm 4.4$ vs. $50.6 \pm 5.4 \mathrm{~ms}, P=0.027)$ and tibial cartilage $(37.1 \pm 6.5$ vs. $42.5 \pm 4.2 \mathrm{~ms}, P=0.042)$. In contrast, no statistically significant increases of the T2 values were observed in the lateral compartments of group II. In group I, neither the medial nor the lateral compartments showed a statistically significant $\mathrm{T} 2$ value increase.

When comparing B with F T2 values subdivided according to the ROIs, group II showed statistically significant increases in the ROIs 3, 4, 6, 7, and 8 of the medial part and in the ROIs 4 and 6 of the lateral part. These results are depicted in Figures 2 and 3. In contrast, in group I, none of 


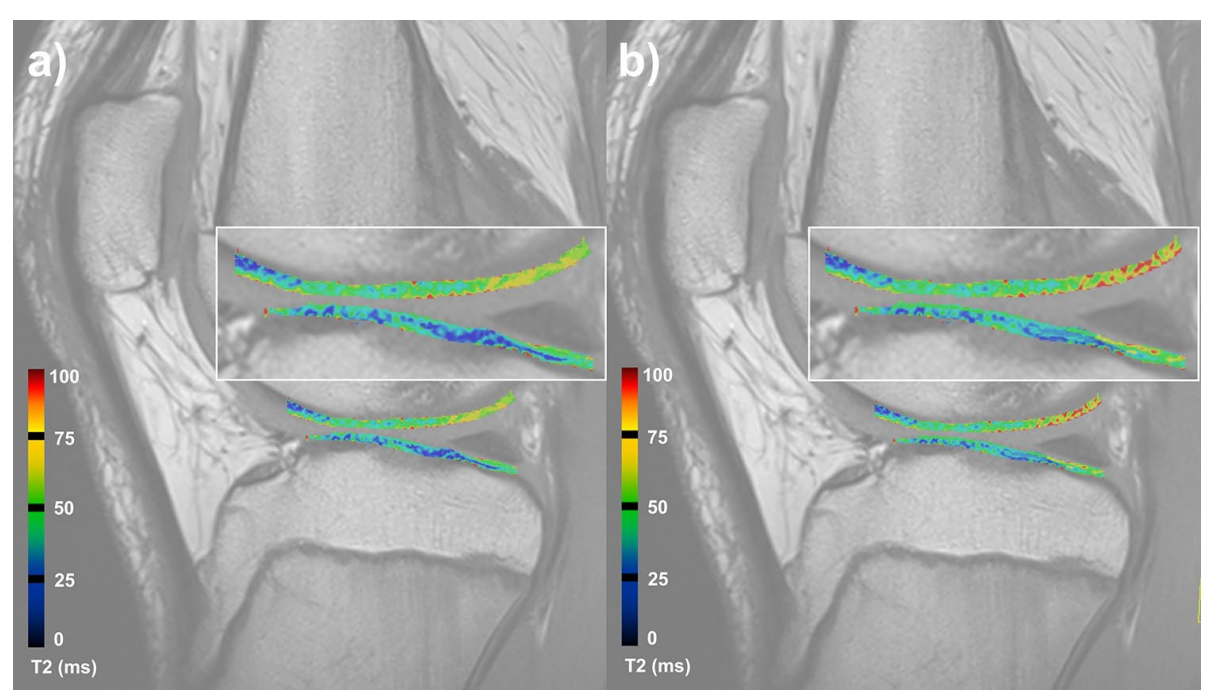

Figure 2. Quantitative sagittal color-coded T2 map of the medial compartment of the knee joint from the baseline investigation (a). The corresponding color-coded T2 map from the follow-up investigation are shown in (b). The areas showing a statistically significant increase of T2 relaxation times in the posterior femoral condyle and posterior tibial plateau (red and yellow), can be clearly seen in this color-coded T2 map.

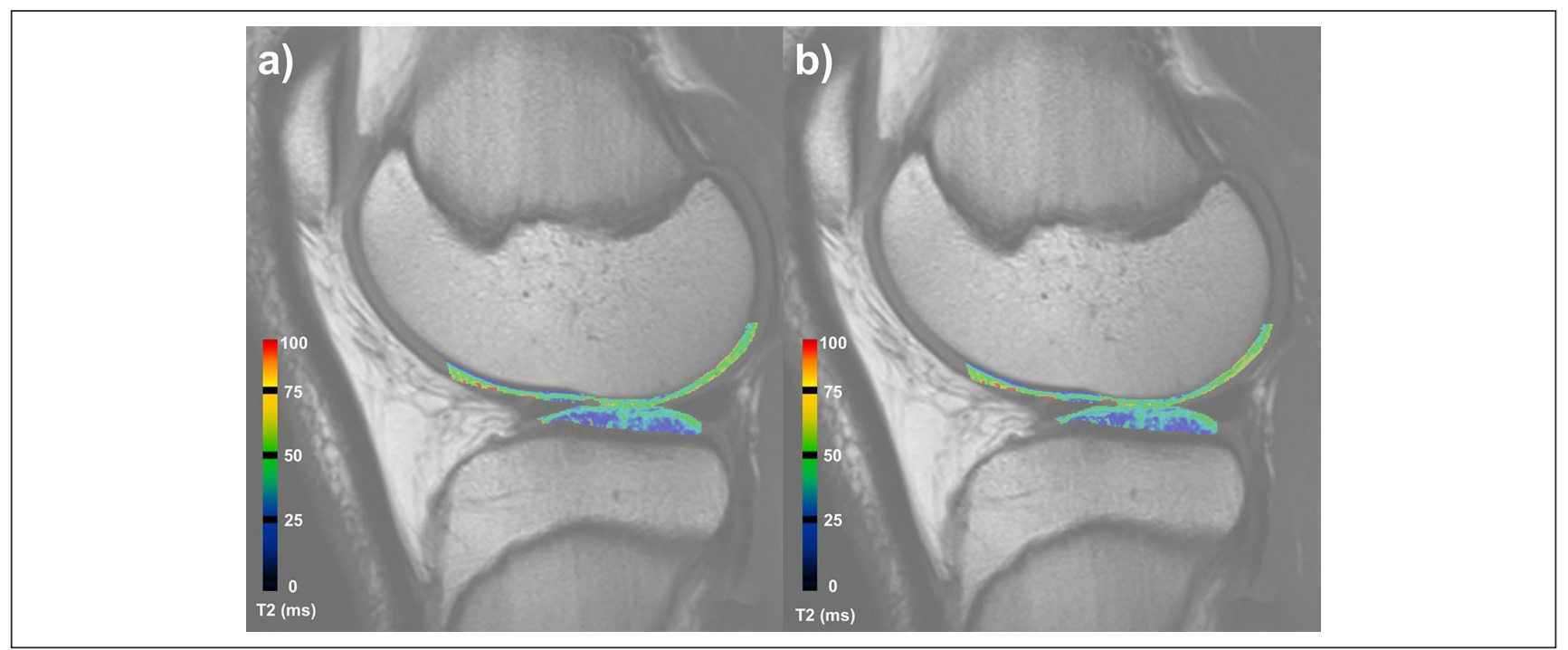

Figure 3. Quantitative sagittal color-coded T2 map of the lateral compartment of the knee joint from the baseline (a) and the follow-up (b) investigation. In (b), there can be seen slightly increased T2 relaxation times in the posterior femoral condyle (yellow) and anterior tibial plateau (green).

the ROIs showed statistically significant increases. In both groups a statistically significant decrease of the T2 values was observed in the ROI 1 of the lateral condyle. These data are summarized in Table 3.

\section{Clinical Assessment}

The Lysholm score reached a mean value of 100 at the B examination and a mean value of $96.3 \pm 6.3$ at the $\mathrm{F}$ examination.
The clinical examination of the supporting leg showed no abnormalities in effusion, range of motion, or ligament stability in any of the players.

\section{Inter- and Intraobserver Intraclass Reliability}

The analysis of inter- and intraobserver reproducibility of our T2 measurements procedure showed an ICC $>0.8$ at the $\mathrm{B}$ and the $\mathrm{F}$ measurements. 
Table 3. T2 Relaxation Times (Mean \pm SD) for All Single Anatomical Regions of Interest (ROls) of Group I (Number of Years Playing Soccer $<15$ Years, $n=7$ ) and Group II (Number of Years Playing Soccer $>15$ Years, $n=12$ ). ${ }^{a}$

\begin{tabular}{|c|c|c|c|c|c|c|c|c|}
\hline \multirow{2}{*}{\multicolumn{2}{|c|}{ Localization }} & & \multicolumn{3}{|c|}{ Group I } & \multicolumn{3}{|c|}{ Group II } \\
\hline & & & $\begin{array}{c}\text { Baseline T2 } \\
\text { (ms) }\end{array}$ & $\begin{array}{l}\text { Follow-up } \\
\text { T2 (ms) }\end{array}$ & $\begin{array}{c}P \text { Value } \\
\text { Difference }\end{array}$ & $\begin{array}{l}\text { Baseline T2 } \\
\text { (ms) }\end{array}$ & $\begin{array}{l}\text { Follow-up } \\
\text { T2 (ms) }\end{array}$ & $\begin{array}{c}P \text { Value } \\
\text { Difference }\end{array}$ \\
\hline \multirow[t]{8}{*}{ Medial } & ROI I & Anterior deep femoral & $39.3 \pm 7.1$ & $43.7 \pm 15.1$ & 0.578 & $36.0 \pm 8.1$ & $40.2 \pm 11.9$ & 0.241 \\
\hline & ROI 2 & Anterior superficial femoral & $60.3 \pm 6.9$ & $67.3 \pm 10.6$ & 0.297 & $53.1 \pm 8.2$ & $60.4 \pm 7.5$ & 0.052 \\
\hline & ROI 3 & Posterior deep femoral & $40.5 \pm 9.8$ & $39.2 \pm 10.7$ & 0.813 & $36.1 \pm 5.4$ & $39.5 \pm 6.2$ & $0.00 \mathrm{I}$ \\
\hline & ROI 4 & Posterior superficial femoral & $69.7 \pm 12.2$ & $69.5 \pm 11.9$ & I & $57.0 \pm 7.6$ & $62.4 \pm 8.3$ & 0.034 \\
\hline & ROI 5 & Anterior deep tibial & $39.0 \pm 4.0$ & $34.0 \pm 7.3$ & 0.219 & $31.9 \pm 8.1$ & $30.2 \pm 3.4$ & 0.791 \\
\hline & ROI 6 & Anterior superficial tibial & $50.3 \pm 5.5$ & $56.1 \pm 7.1$ & 0.578 & $45.0 \pm 8.1$ & $50.5 \pm 6.3$ & 0.034 \\
\hline & ROI 7 & Posterior deep tibial & $32.7 \pm 3.1$ & $33.3 \pm 5.4$ & 0.719 & $28.3 \pm 4.7$ & $34.1 \pm 5.7$ & 0.009 \\
\hline & ROI 8 & Posterior superficial tibial & $51.7 \pm 3.7$ & $53.0 \pm 5.8$ & 0.813 & $43.2 \pm 7.0$ & $55.3 \pm 7.9$ & 0.002 \\
\hline \multirow[t]{8}{*}{ Lateral } & ROI I & Anterior deep femoral & $37.6 \pm 7.0$ & $29.4 \pm 5.4$ & 0.031 & $32.0 \pm 10.4$ & $25.6 \pm 6.7$ & 0.02 \\
\hline & ROI 2 & Anterior superficial femoral & $55.4 \pm 9.0$ & $52.2 \pm 8.4$ & 0.813 & $45.1 \pm 9.2$ & $44.8 \pm 7.0$ & I \\
\hline & ROI 3 & Posterior deep femoral & $49.2 \pm 15.7$ & $53.1 \pm 14.2$ & 0.813 & $43.3 \pm 6.3$ & $46.9 \pm 8.6$ & 0.11 \\
\hline & ROI 4 & Posterior superficial femoral & $79.2 \pm 12.3$ & $81.8 \pm 18.6$ & 0.578 & $61.9 \pm 8.0$ & $72.4 \pm 7.7$ & 0.007 \\
\hline & ROI 5 & Anterior deep tibial & $30.1 \pm 6.2$ & $27.3 \pm 3.7$ & 0.219 & $26.0 \pm 2.9$ & $25.6 \pm 2.2$ & 0.677 \\
\hline & ROI 6 & Anterior superficial tibial & $44.3 \pm 11.7$ & $46.9 \pm 5.5$ & 0.813 & $35.7 \pm 3.6$ & $41.6 \pm 6.6$ & 0.016 \\
\hline & ROI 7 & Posterior deep tibial & $29.5 \pm 4.2$ & $30.6 \pm 7.0$ & 0.813 & $28.1 \pm 7.3$ & $30.5 \pm 7.8$ & 0.151 \\
\hline & ROI 8 & Posterior superficial tibial & $47.3 \pm 8.8$ & $55.8 \pm 13.7$ & 0.219 & $45.8 \pm 9.0$ & $51.4 \pm 10.9$ & 0.052 \\
\hline
\end{tabular}

${ }^{a}$ The $T 2$ values are displayed for the baseline (B) and the follow-up (F) examination with the calculated $P$ value for the difference. $P$ values $\leq 0.05$ are marked in boldface.

\section{Discussion}

The aim of our longitudinal study was to investigate the tibiofemoral joint cartilage of adolescent professional soccer players with regard to early cartilage degenerations in a $\mathrm{B}$ and an $\mathrm{F}$ investigation 4,3 years later. We hypothesized that the high mechanical load on the knee joint of soccer players affects the cartilage structure during adolescence.

Our results suggest that the medial cartilage regions, especially the medial posterior zones, are at risk for early degenerative changes. Knowing these regions can help to optimize training and prevention programs to avoid the development of these early alterations. Quantitative T2 mapping was used to assess changes in the cartilage matrix. The elevation of $\mathrm{T} 2$ values in the global medial compartments and in the subregional medial posterior areas of the knee joint indicates that loading forces in these areas are relevant in soccer.

Our examination demonstrated increases in the $\mathrm{T} 2$ relaxation times in the cartilage of the whole cohort from the $\mathrm{B}$ to the F observation. Especially a statistically significant increase in the medial compartment could be detected. These findings support the hypothesis that intensive physical activity like soccer can influence the cartilage structure already at the time of adolescence. All players had excellent Lysholm scores during the observation. They were healthy and actively playing soccer. T2 mapping can be seen as a marker for tissue alterations at a very early stage as $\mathrm{T} 2$ changes occur before the onset of clinical subjective or objective symptoms. Quantitative T2 mapping is a noninvasive assessment tool that shows early cartilage changes. Elevated $\mathrm{T} 2$ values indicate damaged cartilage compared to healthy cartilage. ${ }^{19,20}$ In our cohort the increase of the T2 values over time can partly be seen as an adaptation to the high biomechanical loads and additionally as the early onset of degenerative changes. Several investigators studied the influence of soccer on OA. ${ }^{5,21}$ Overall more pronounced changes in the radiographic and the MR images of the knee joint were found in the former soccer players compared with non-professional athletes. ${ }^{11}$ Our presented results of a statistically significant increase of the $\mathrm{T} 2$ values in the medial compartment of the tibiofemoral joint underscore that the biomechanical load is highest on the medial condyle. ${ }^{22-24} \mathrm{~T} 2$ values showed the highest change from early to late unloading in the medial femoral condyle, suggesting the cartilage of the medial condyle is more affected by degeneration. ${ }^{25}$ Similar observations were also made in previously published studies. ${ }^{26,27}$ According to several studies reviewing chondral defects by knee arthroscopies, the most common cartilage lesions were localized on the medial femoral condyle. ${ }^{28-30}$

The T2 changes were more pronounced in the group playing soccer for more than 15 years (group II). In this group, a statistically significant increase of the T2 values was found in the pooled ROIs for the medial compartment. Whereas in the group with less than 15 years (group I) 
active playing time no statistically significant changes were detected. We conclude that the longer the athlete has been exposed to training stress, the more pronounced is the cartilage alteration. The dependency between age and skeletal maturation has been described in a study by Kim et al. ${ }^{31}$ They postulated that an increase in $\mathrm{T} 2$ relaxation times in children could indicate abnormal cartilage reaction independent of the skeletal maturation. Hence our results could indicate that the intense activity in our investigated athletes has influenced the cartilage composition. The alterations of the cartilage structure begin superficially near the articular surface and will proceed to the deep layer in a more advanced stage..$^{20,32}$

The results of the subregional analysis suggest that especially the medial posterior part of the knee joint is more likely to be influenced by loading and might result with a more sensitive reaction. Our analysis showed statistically significant increases of the $\mathrm{T} 2$ values over time in the superficial and even in the deep layer in the posterior areas of the medial compartment. This was conspicuous in group II for the femoral and the tibial ROIs and in the whole cohort for the tibial ROIs. In contrast, the anterior zones and lateral compartments showed no statistically significant changes or only in the superficial layers. Our examination focused on the non-dominant leg. During kicking it is in flexion and the mechanical load increases in the posterior part of the knee joint. The detected increases of the $\mathrm{T} 2$ relaxation times in the posterior superficial and deep layer reflect the influence of these loading forces on the cartilage. The contact pressure shifts posteriorly when the knee joint reaches a flexion of $30^{\circ}{ }^{33}$ This observation was confirmed by Brown et al. ${ }^{22}$ Chen et al..$^{34}$ observed the effect of walking, running, and stair activity on the knee articular cartilage using T2 mapping. Their results showed the highest T2 changes in the posterior weightbearing regions of the tibiofemoral joint. They supposed that higher loading and friction at these regions leads to more vulnerability for cartilage damage.

Our study will be one of the necessary steps toward the possibility to specifically identify anatomical vulnerable cartilage regions. The results might give hints for the development of focused programs for soccer training in general. Prevention of OA in professional soccer players is very important, and this has been discussed in several recent studies. ${ }^{35-38}$ It is conceivable that based on T2 mapping personalized treatment programs and prevention programs can be constructed already at the time of adolescence. Identify the region at risk may help create an adequate training program with those exercises, which reduce the mechanical load on these vulnerable areas. Strengthening of the supporting muscles, stabilization of the leg axis, improvement of the proprioception and optimizing the sensorimotor control should be ensured. Individual exercises for each player may be established. Treadmill analysis of the players' motion pattern can play a role and helps develop individualized equipment, for example shoes with orthopedic insoles, which can decrease joint loading and circumscribed impact on cartilage areas. Adequate training during adolescence plays an important role in maintaining the health of professional soccer player and to prevent the progress to OA.

There are some limitations and open questions of the presented study. Longer F periods and larger cohorts will be necessary to confirm the results. In a larger cohort, it could be possible to define clear cutoff T2 values for the definition of degenerative changes in young and active athletes. The interpretation of T2 mapping results may be simplified in clinical routine. Based on cutoff values, cartilage screening tools by $\mathrm{T} 2$ mapping can be established and may become routine in the medical monitoring of professional sport teams. Understandably, it was not feasible to obtain histological or surgical proof as gold standard of our results.

The $\mathrm{T} 2$ values increase with advanced cartilage degeneration. The identification of cartilage with early alterations will be our aim in order to improve prevention and treatment in sports. Our results showed several vulnerable areas with increased $\mathrm{T} 2$ values in the knee joint in young professional soccer players. This pattern might give a hint that the impact of high-intensity training on the knee joint influence cartilage structure as early as at the time of adolescence and could be a risk factor for developing knee OA. The development of specific training concepts is important to avoid these early cartilage alterations. Injury prevention concepts in soccer are currently being established. The FIFA $11+$ kids program provides a good benefit, which improves physical performance parameters followed by a reduction of soccerrelated injuries. ${ }^{39}$ It is conceivable that specific training exercises, which decrease the impact loading in the vulnerable areas, can optimize the prevention programs. However, there are still many aspects that have to be kept in mind. Our results are a first step toward individualized risk assessment for young sports professionals not only in soccer and may improve injury prevention and training programs.

\section{Conclusion}

In conclusion, early cartilage degenerations can be detected by T2 mapping in the knee joint of young professional soccer players. The medial cartilage regions, especially the medial posterior zones, seem to be at risk. The high mechanical load affects the cartilage structure already at the time of adolescence. Knowing the pattern of the most vulnerable regions is useful to generate adequate training concepts and prevention programs for adolescent soccer players to minimize the early cartilage alterations in these vulnerable regions and hence to avoid pre-osteoarthritis. Individualized training programs should play a role in maintaining the health of professional soccer players, to avoid the incidence of OA in the middle-aged with following reduced quality of life and T2 mapping can be part of subtle and individual diagnosis programs. 


\section{Authors' Note}

The present work was done at Department of Trauma and Orthopedic Surgery, University Hospital Erlangen of the FriedrichAlexander-University Erlangen-Nürnberg (FAU), Germany. The present work was performed in fulfillment of the requirements for obtaining the degree "Dr. med."

\section{Acknowledgments and Funding}

We wish to thank all volunteers who took part in this study for their participation. The author(s) disclosed receipt of the following financial support for the research, authorship, and/or publication of this article: This project was in parts supported by the Interdisciplinary Center for Clinical Research (IZKF) at the University Hospital of the University of Erlangen-Nürnberg (FAU) (ELAN P005) and by the Emerging Fields Initiative (EFI) of the FAU and the STAEDTLER Stiftung (EFI_Verbund Med 05 MIRACLE to MLP). Sources of funding had no role or input in study design, collection, analysis, and interpretation of data, in writing of the article, and in the decision to submit the article for publication.

\section{Declaration of Conflicting Interests}

The author(s) declared the following potential conflicts of interest with respect to the research, authorship, and/or publication of this article: Rolf Janka has received payment for lectures from Siemens Healthineers and Bracco Imaging. All other authors declared no potential conflicts of interest.

\section{Ethical Approval}

All procedures performed in studies involving human participants were in accordance with the ethical standards of the institutional and/or national research committee and with the 1964 Helsinki declaration and its later amendments or comparable ethical standards. Ethical approval for this study was obtained from the ethics committee of the Friedrich-Alexander-University ErlangenNürnberg (FAU), Germany (approval number: 4397).

\section{Informed Consent}

Written informed consent was obtained from all individual participants included in the study.

\section{Trial Registration}

Not applicable.

\section{ORCID iDs}

Christoph Evers (iD https://orcid.org/0000-0002-7115-474X

Milena L. Pachowsky iD https://orcid.org/0000-0002-3729-9566

\section{References}

1. Junge A, Dvorak J. Injury surveillance in the World Football Tournaments 1998-2012. Br J Sports Med. 2013;47:782-8.

2. O'Kane JW, Neradilek M, Polissar N, Sabado L, Tencer A, Schiff MA. Risk factors for lower extremity overuse injuries in female youth soccer players. Orthop J Sports Med. 2017; 5(10):2325967117733963. doi:10.1177/2325967117733963
3. Roemer FW, Jarraya M, Niu J, Silva JR, Frobell R, Guermazi A. Increased risk for radiographic osteoarthritis features in young active athletes: a cross-sectional matched case-control study. Osteoarthritis Cartilage. 2015;23:239-43.

4. Lee HH, Chu CR. Clinical and basic science of cartilage injury and arthritis in the football (soccer) athlete. Cartilage. 2012;3(1 Suppl):63S-68S.

5. Paxinos O, Karavasili A, Delimpasis G, Stathi A. Prevalence of knee osteoarthritis in 100 athletically active veteran soccer players compared with a matched group of 100 military personnel. Am J Sports Med. 2016;44:1447-54.

6. Felson DT, Lawrence RC, Dieppe PA, Hirsch R, Helmick CG, Jordan JM, et al. Osteoarthritis: new insights. Part 1: the disease and its risk factors. Ann Intern Med. 2000;133:635-46.

7. Roos H, Lindberg H, Gardsell P, Lohmander LS, Wingstrand $H$. The prevalence of gonarthrosis and its relation to meniscectomy in former soccer players. Am J Sports Med. 1994;22:219-22.

8. Amano K, Pedoia V, Su F, Souza RB, Li X, Ma CB. Persistent biomechanical alterations after ACL reconstruction are associated with early cartilage matrix changes detected by quantitative MR. Orthop J Sports Med. 2016;4(4):2325967116644421. doi:10.1177/2325967116644421

9. Gouttebarge V, Aoki H, Kerkhoffs GMMJ. Knee osteoarthritis in professional football is related to severe knee injury and knee surgery. Inj Epidemiol. 2018;5:26.

10. Hofmann FC, Neumann J, Heilmeier U, Joseph GB, Nevitt $\mathrm{MC}, \mathrm{McCulloch} \mathrm{CE}$, et al. Conservatively treated knee injury is associated with knee cartilage matrix degeneration measured with MRI-based T2 relaxation times: data from the osteoarthritis initiative. Skeletal Radiol. 2018;47:93-106.

11. Arliani GG, Astur DC, Yamada RK, Yamada AF, Miyashita GK, Mandelbaum B, et al. Early osteoarthritis and reduced quality of life after retirement in former professional soccer players. Clinics (Sau Paulo). 2014;69:589-94.

12. Ebert JR, Robertson WB, Woodhouse J, Fallon M, Zheng $\mathrm{MH}$, Ackland T, et al. Clinical and magnetic resonance imaging-based outcomes to 5 years after matrix-induced autologous chondrocyte implantation to address articular cartilage defects in the knee. Am J Sports Med. 2011;39:753-63.

13. Bittersohl B, Hosalkar HS, Miese FR, Schibensky J, König DP, Herten M, et al. Zonal T2* and T1Gd assessment of knee joint cartilage in various histological grades of cartilage degeneration: an observational in vitro study. BMJ Open. 2015;5(2):e006895. doi:10.1136/bmjopen-2014-006895

14. Eagle S, Potter HG, Koff MF. Morphologic and quantitative magnetic resonance imaging of knee articular cartilage for the assessment of post-traumatic osteoarthritis. J Orthop Res. 2017;35:412-23.

15. Liess C, Lusse S, Karger N, Heller M, Gluer CC. Detection of changes in cartilage water content using MRI T2-mapping in vivo. Osteoarthritis Cartilage. 2002;10:907-13.

16. Trattnig S, Mamisch TC, Welsch GH, Glaser C, Szomolanyi $\mathrm{P}$, Gebetsroither $\mathrm{S}$, et al. Quantitative T2 mapping of matrixassociated autologous chondrocyte transplantation at 3 Tesla: an in vivo cross-sectional study. Invest Radiol. 2007;42:442-8.

17. Apprich S, Welsch GH, Mamisch TC, Szomolanyi P, Mayerhoefer M, Pinker K, et al. Detection of degenerative cartilage disease: comparison of high-resolution morphological 
MR and quantitative T2 mapping at 3.0 Tesla. Osteoarthritis Cartilage. 2010;18:1211-7.

18. Liebl H, Joseph G, Nevitt MC, Singh N, Heilmeier U, Subburaj K, et al. Early T2 changes predict onset of radiographic knee osteoarthritis: data from the osteoarthritis initiative. Ann Rheum Dis. 2015;74:1353-9.

19. Soellner ST, Goldmann A, Muelheims D, Welsch GH, Pachowsky ML. Intraoperative validation of quantitative T2 mapping in patients with articular cartilage lesions of the knee. Osteoarthritis Cartilage. 2017;25:1841-9.

20. Baum T, Joseph GB, Karampinos DC, Jungmann PM, Link TM, Bauer JS. Cartilage and meniscal T2 relaxation time as non-invasive biomarker for knee osteoarthritis and cartilage repair procedures. Osteoarthritis Cartilage. 2013;21:1474-84.

21. Krajnc Z, Vogrin M, Recnik G, Crnjac A, Drobnic M, Antolic $\mathrm{V}$. Increased risk of knee injuries and osteoarthritis in the nondominant leg of former professional football players. Wien Klin Wochenschr. 2010;122(Suppl 2):40-3.

22. Brown TD, Shaw DT. In vitro contact stress distribution on the femoral condyles. J Orthop Res. 1984;2:190-9.

23. Van Rossom S, Smith CR, Zevenbergen L, Thelen DG, Vanwanseele B, Van Assche D, et al. Knee cartilage thickness, $\mathrm{T} 1 \rho$ and $\mathrm{T} 2$ relaxation time are related to articular cartilage loading in healthy adults. PLoS One. 2017;12(1):e0170002. doi:10.1371/journal.pone.0170002

24. Walker PS, Hajek JV. The load-bearing area in the knee joint. J Biomech. 1972;5:581-9.

25. Waldenmeier L, Evers C, Uder M, Janka R, Hennig FF, Pachowsky ML, et al. Using cartilage MRI T2-mapping to analyze early cartilage degeneration in the knee joint of young professional soccer players. Cartilage. 2018;10(3):288-98. doi:10.1177/1947603518756986

26. Luke AC, Stehling C, Stahl R, Li X, Kay T, Takamoto S, et al. High-field magnetic resonance imaging assessment of articular cartilage before and after marathon running: does long-distance running lead to cartilage damage? Am J Sports Med. 2010;38:2273-80.

27. Subburaj K, Kumar D, Souza RB, Alizai H, Li X, Link TM, et al. The acute effect of running on knee articular cartilage and meniscus magnetic resonance relaxation times in young healthy adults. Am J Sports Med. 2012;40:2134-41.
28. Aroen A, Loken S, Heir S, Alvik E, Ekeland A, Granlund OG, et al. Articular cartilage lesions in 993 consecutive knee arthroscopies. Am J Sports Med. 2004;32:211-5.

29. Hjelle K, Solheim E, Strand T, Muri R, Brittberg M. Articular cartilage defects in 1000 knee arthroscopies. Arthroscopy. 2002;18:730-4.

30. Curl WW, Krome J, Gordon ES, Rushing J, Smith BP, Poehling GG. Cartilage injuries: a review of 31,516 knee arthroscopies. Arthroscopy. 1997;13:456-60.

31. Kim HK, Shiraj S, Anton CG, Horn PS, Dardzinski BJ. Age and sex dependency of cartilage T2 relaxation time mapping in MRI of children and adolescents. AJR Am J Roentgenol. 2014;202:626-32.

32. Cha JG, Lee JC, Kim HJ, Han JK, Lee EH, Kim YD, et al. Comparison of MRI T2 relaxation changes of knee articular cartilage before and after running between young and old amateur athletes. Korean J Radiol. 2012;13:594-601.

33. Cuellar VG, Martinez D, Immerman I, Oh C, Walker PS, Egol KA. A biomechanical study of posteromedial tibial plateau fracture stability: do they all require fixation? J Orthop Trauma. 2015;29:325-30.

34. Chen M, Qiu L, Shen S, Wang F, Zhang J, Zhang C, et al. The influences of walking, running and stair activity on knee articular cartilage: quantitative MRI using T1 rho and T2 mapping. PLoS One. 2017;12(11):e0187008. doi:10.1371/journal.pone.0187008

35. Gouttebarge V, Inklaar H, Frings-Dresen MH. Risk and consequences of osteoarthritis after a professional football career: a systematic review of the recent literature. J Sports Med Phys Fitness. 2014;54:494-504.

36. Salzmann GM, Preiss S, Zenobi-Wong M, Harder LP, Maier D, Dvorak J. Osteoarthritis in football. Cartilage. 2017;8:162-72.

37. Kiani A, Hellquist E, Ahlqvist K, Gedeborg R, Michaelsson $\mathrm{K}$, Byberg L. Prevention of soccer-related knee injuries in teenaged girls. Arch Intern Med. 2010;170:43-9.

38. Kuijt MT, Inklaar H, Gouttebarge V, Frings-Dresen MH. Knee and ankle osteoarthritis in former elite soccer players: a systematic review of the recent literature. J Sci Med Sport. 2012;15:480-7.

39. Pomares-Noguera C, Ayala F, Robles-Palazón FJ, AlomotoBurneo JF, López-Valenciano A, Elvira JLL, et al. Training effects of the FIFA $11+$ kids on physical performance in youth football players: a randomized control trial. Front Pediatr. 2018;6:40. doi:10.3389/fped.2018.00040 